\title{
Vermicompostaje del bagazo de uva: fuente de enmienda orgánica de alta calidad agrícola y de polifenoles bioactivos
}

Recibido: 9 outubro 2013 / Aceptado: 25 novembro 2013

(C) IBADER- Universidade de Santiago de Compostela 2013
Resumen El bagazo de uva es un residuo vitivinícola generado en grandes cantidades en regiones como Galicia (España). Posee unas concentraciones polifenólicas elevadas que inhiben el crecimiento de raíces y plantas y son un riesgo para su aplicación como enmienda orgánica en agricultura sin un proceso de tratamiento previo. Pero los polifenoles son también compuestos bioactivos naturales, y por su carácter antioxidante y demás propiedades asociadas son sustancias a considerar en la obtención de ingredientes para la industria cosmética, farmacéutica o alimentaria. En este experimento se realizó un proceso de vermicompostaje del bagazo de uva de la variedad Albariño y se estudiaron los principales cambios físicos, biológicos y químicos (particularmente, polifenólicos) que se producen durante su degradación. Tras 42 días de vermicompostaje, las semillas aisladas presentaban todavía unas concentraciones de polifenoles importantes y de elevado interés industrial. En cuanto al bagazo vermicompostado las concentraciones polifenólicas disminuyeron rápidamente hasta el final del proceso (día 112) y se obtuvo un vermicompost final maduro, estable y de calidad para su uso como enmienda orgánica en campos de cultivo.

Palabras clave Residuo vitivinícola, compuestos polifenólicos, vermirreactor, lombriz de tierra, vermicompost.

Hugo Martínez-Cordeiro · Jorge Domínguez

Departamento de Ecoloxía e Bioloxía Animal, Universidade de

Vigo, E-36310 Vigo

Tel: 699582447

E-mail: hmartinez@uvigo.es

Marta Álvarez-Casas · Marta Lores

Departamento de Química Analítica, Nutrición e Bromatoloxía, Universidade de Santiago de Compostela, E-15782 Santiago de Compostela
Abstract Grape marc is a winery waste generated in large quantities in regions such as Galicia (Spain). It has high polyphenol concentrations that inhibit the growth of roots and plants and that could become a risk in using it as organic amendment in agriculture without pretreatment process. Polyphenols are natural bioactive compounds that for their antioxidant properties and other associated properties are important substances considered in obtaining ingredients for cosmetic, pharmaceutical or food industry. In this experiment we performed a vermicomposting process with grape marc from the Albariño variety. The main physical, biological and chemical changes (particularly, polyphenolics) during the degradation process have been studied. After 42 days of vermicomposting, the isolated seeds still have high concentrations of polyphenols with important industrial interest. The polyphenolic content of the vermicomposted grape marc was decreasing rapidly until the end of the process (day 112); and a mature, stable and high quality vermicompost was obtained for use as organic fertilizer on crops.

Key words Winery waste, polyphenolic compounds, vermirreactor, earthworm, vermicompost.

\section{Introducción}

El bagazo de uva es un subproducto de la industria vitivinícola que se obtiene tras los procesos de extracción del mosto en la preparación del vino. Está formado por los tallos, piel, pulpa, semillas y racimos que quedan después del prensado de la uva. Aproximadamente, de cada $100 \mathrm{~kg}$ de uva procesada se obtienen entre 15 y $20 \mathrm{~kg}$ de residuo (Usaquén-Castro et al. 2006). Se estima que a nivel mundial la industria vitivinícola produce una cantidad de al menos 10 millones de toneladas anuales de residuo (Maier et al. 2009), de las cuales en España se producen alrededor de 1 millón de toneladas al año. Se trata de un sustrato valioso debido a un alto contenido en nutrientes, principalmente nitrógeno y potasio, esenciales para el crecimiento de plantas y desarrollo de cultivos (Bertran et al. 2004) y posee cantidades importantes de lignina, celulosa y polifenoles 
(Dell'Agli et al. 2004). En la actualidad, la principal aplicación del bagazo es su utilización como enmienda orgánica en los campos de cultivo, pero sin un proceso de tratamiento previo (Bustamante 2007; Cegarra \& Paredes 2007). Esto produce la liberación de polifenoles en el suelo los cuales pueden inhibir el crecimiento de las raíces (Inbar et al. 1991) y en consecuencia, afectar al desarrollo de las plantas, pudiendo ser perjudicial en su uso cómo enmienda orgánica en campos de cultivo. Los polifenoles constituyen uno de los grupos de metabolitos secundarios más numeroso y ampliamente distribuido en el reino vegetal (Sardesai 1995). Son compuestos bioactivos naturales (Gharras 2009; Quideau et al. 2011) y por su carácter antioxidante y demás propiedades asociadas son sustancias a considerar en la obtención de ingredientes para la industria cosmética, farmacéutica o alimentaria. Los efectos beneficiosos de los polifenoles se atribuyen principalmente a sus propiedades antioxidantes, ya que pueden actuar, dependiendo de su estructuras químicas, como eliminadores de radicales libres (Rice-Evans 2001), los cuales están directamente involucrados en fenómenos de estrés oxidativo y en numerosas patologías asociadas.

Una alternativa de tratamiento del bagazo es mediante un proceso de vermicompostaje. Este trabajo demuestra que dicho proceso facilita la obtención de compuestos polifenólicos que, de modo simultáneo, se aprovechan para su uso a nivel industrial y se eliminan del producto final, con el fin de mejorar su calidad como enmienda orgánica en campos de cultivo. Los sistemas de vermicompostaje sostienen una compleja cadena trófica donde las lombrices de tierra detritívoras y principalmente epigeas interactúan de forma intensa con los microorganismos y demás fauna descomponedora. Se trata de un proceso de biooxidación, degradación y estabilización de los sustratos orgánicos mediante dos fases, una fase inicial o activa en la cual las lombrices acondicionan y fragmentan el sustrato incrementando el área expuesta para los microorganismos (Domínguez et al. 2010) y una fase de maduración en la cual los microorganismos más especializados asumen el control del proceso y degradan compuestos más complejos (Domínguez et al. 2010).

Las lombrices promueven una separación mecánica del sustrato, trasformando las partes mejor asimilables en un vermicompost ya estabilizado, homogéneo, rico en nutrientes y de granulometría fina (Domínguez, 2004) que ocupa las capas inferiores. De esta manera, aumenta la concentración de semillas en las capas superficiales y se facilita así el proceso de tamizado que tiene lugar una vez finalizado el vermicompostaje. Estas semillas las partes de mayor concentración polifenólica y por lo tanto de elevado interés para usos industriales.

En general, el proceso de vermicompostaje es una tecnología rápida y de bajo coste para la gestión de residuos poco valorados de diferente naturaleza (Domínguez \& Edwards, 2010) y por lo tanto, potencialmente adecuado para su uso con bagazo de uva. Con este proceso no solo se favorece su eliminación, sino que también se ofrece una alternativa económica adicional y nuevas oportunidades para el uso de este subproducto.
En función de estas características, el objetivo de este experimento consiste en realizar un estudio completo y detallado sobre el potencial del vermicompostaje como tratamiento del bagazo de uva, la posible bioconversión de este subproducto en enmienda orgánica para horticultura y como fuente de compuestos polifenólicos bioactivos de interés industrial concentrados principalmente en las semillas.

\section{Materiales y métodos}

\section{Bagazo de uva}

El bagazo de uva utilizado estaba constituido por tallos, piel, pulpa, semillas y racimos y pertenecía a la variedad Albariño (Vitis vinifera), cultivada en Galicia para la producción de vinos blancos de alta calidad bajo la Denominación de Origen Rías Baixas. El bagazo fue proporcionado por la bodega Mar de Frades situada en San Martiño de Meis, (Pontevedra) y se almacenó en una cámara fría a una temperatura de $4^{\circ} \mathrm{C}$ hasta su utilización.

\section{Especie de lombriz utilizada}

Se utilizaron lombrices de tierra de la especie Eisenia andrei Bouché (1972) pertenecientes a la categoría ecológica de epigeas. Estas lombrices viven en el horizonte orgánico del suelo y son de pequeño tamaño, pigmentadas, con una alta tasa reproductiva, ciclo de vida corto, alta tasa de consumo y una gran asimilación de la materia orgánica en descomposición.

\section{Funcionamiento del vermirreactor}

El procesamiento del bagazo tuvo lugar en un vermirreactor de una superficie de $6 \mathrm{~m}^{2}(1,5 \times 4 \mathrm{~m})$ con una cama de vermicompost maduro para asegurar la supervivencia de las lombrices y una población ya presente de lombrices de tierra Eisenia andrei de $214 \pm 26$ individuos $\mathrm{m}^{-2}$. Las lombrices desmenuzan el residuo en las capas superficiales y se descarga el vermicompost en la parte inferior a través de un suelo perforado.

Antes de introducir el bagazo en el vermirreactor se procedió a airearlo y humedecerlo durante 4 días a temperatura ambiente para conseguir una humedad óptima para las lombrices, en torno al $80-85 \%$. Sobre la superficie de la cama de vermicompost y para separarlo del bagazo de uva se dispuso una red de plástico de $5 \mathrm{~mm}$ de luz de malla que permitió el paso de las lombrices a su través sin afectar de este modo al procesado del residuo y para facilitar la toma de muestras durante el proceso de vermicompostaje. Una vez introducido el bagazo en el vermirreactor, se extendió sobre la red de manera homogénea y se cubrió con una malla de sombra para reducir la evaporación de agua y mantener una humedad óptima para la supervivencia de las lombrices. 


\section{Población de lombrices}

Para el seguimiento de la población de lombrices se recogieron, cada 14 días, 5 muestras que contenían cada una un espesor de $6 \mathrm{~cm}$ recogidos por encima de la malla donde estaba el residuo y $6 \mathrm{~cm}$ por debajo en el mismo sitio correspondiente a la zona de la cama. Las muestras se recogieron con un extractor cilíndrico $(7,5 \mathrm{~cm}$ de diámetro y $12 \mathrm{~cm}$ de altura) a lo largo de los 112 días del proceso de vermicompostaje.

\section{Toma de muestras}

Para el análisis de las características físicas, químicas y biológicas se recogieron de manera aleatoria, 5 muestras de 10 gramos de bagazo de uva vermicompostado y 5 muestras de 10 gramos de semillas aisladas manualmente en los días 0,56 y 112 del vermicompostaje. Para el análisis de los polifenoles totales se recogieron de manera aleatoria, y cada 7 días, 5 muestras de 10 gramos de bagazo de uva vermicompostado, las cuales a partir del día 42 fueron tamizadas a través de $1 \mathrm{~mm}$ de luz de malla y se analizó solamente el vermicompost producido; y 5 muestras de 10 gramos de semillas aisladas manualmente durante el proceso de vermicompostaje. Para el análisis de polifenoles individuales presentes en semilla se recogieron de manera aleatoria 5 muestras de 10 gramos de semillas aisladas manualmente en los días 0 y 112 del vermicompostaje. Todas las muestras se almacenaron en bolsas de plástico a una temperatura de $-20^{\circ} \mathrm{C}$ hasta el momento de la realización de los análisis.

\section{Procedimientos analíticos}

Análisis físicos, químicos y biológicos: $\mathrm{El} \mathrm{pH}$ y la conductividad eléctrica se midieron en extractos de agua destilada en proporción 1:10 (peso fresco: volumen); y el contenido de materia orgánica por pérdida de peso tras la calcinación de la muestra seca a $550 \pm 50^{\circ} \mathrm{C}$ durante 4 horas en un horno-mufla. La concentración de $\mathrm{C}$ y $\mathrm{N}$ total se determinó en muestra seca con un analizador elemental CHNS-O EA-1108; y la concentración de $\mathrm{P}$ y $\mathrm{K}$ total en muestras secas sometidas a digestión previa $y$ determinadas mediante Espectrometría de Emisión Óptica con Plasma Acoplado Inductivamente (ICP-OES). La actividad microbiana estimada como respiración basal se realizó a través de un equipo de botellas Oxitop $®$ y el análisis de fibras (celulosa, hemicelulosa y lignina) se realizó según el método fibra detergente propuesto por Goering \& Van Soest (1970).

Extracción de polifenoles: Se utilizó la técnica de extracción con disolventes presurizados (PSE, Pressurized Solvent Extraction) mediante un equipo de PSE comercializado por Dionex (ASE 150). Las muestras se molieron y se extrajeron con un $63 \%$ de disolvente orgánico (metanol) en agua y se concentraron 10 veces para su posterior análisis por cromatografía de líquidos, utilizando un HPLC Varian
Prostar con detector de red de diodos (DAD) y una columna Waters Nova-Pak C18 3,9 x 150 mm.

Determinación de polifenoles totales: Se determinaron por el método de Folin Ciocalteu (Singleton \& Rossi, 1965) mezclando $5 \mathrm{~mL}$ de la muestra diluida en agua milli-Q, 100 $\mu \mathrm{L}$ del reactivo Folin \& Ciocalteu y $1 \mathrm{~mL}$ de una disolución de carbonato sódico (20\% $\mathrm{Na}_{2} \mathrm{CO}_{3}$ en $\mathrm{H}_{2} \mathrm{O}$ Milli-Q). Se agitó en vortex y se dejó reposar 30 minutos a temperatura ambiente y oscuridad para reducir el reactivo Folin \& Ciocalteu por los compuestos polifenólicos. Se realizó la lectura de las absorbancias a una longitud de onda de 760 $\mathrm{nm}$ (espectofotómetro Shimazdu, UVmini-1240, Tokio, Japón) y la concentración de polifenoles totales se calculó mediante una curva de calibrado de ácido gálico. Los resultados fueron expresados como equivalentes de ácido gálico por litro (mg.L-1 GAE).

Identificación y cuantificación de los polifenoles: La identificación de los diferentes polifenoles se llevó a cabo a dos longitudes de onda distintas (280 y $350 \mathrm{~nm}$ ) a las que absorben estos compuestos en función de su estructura. A partir de la biblioteca de espectros UV y con una selección de patrones comerciales de polifenoles se compararon, tanto los espectros como los tiempos de retención de dichos patrones, con los picos cromatográficos obtenidos en la inyección de los extractos de semilla. De esta manera se pudieron identificar los cuatro polifenoles principales en las semillas durante todo el proceso (ácido gálico, catequina, epicatequina y quercetinas) y se determinó la concentración de cada uno a través de las rectas de calibrado de los patrones correspondientes

\section{Análisis estadístico}

Se realizó una prueba t de Student de muestras pareadas para el seguimiento de la población de lombrices, para la caracterización física, química y biológica del bagazo y para la evolución de polifenoles individuales durante el proceso de vermicompostaje. Para la evolución de los polifenoles totales presentes en el bagazo vermicompostado y semillas se utilizó un ANOVA de medidas repetidas y un test a posteriori HSD de Tukey utilizando el paquete estadístico SPSS $\vee 19$

\section{Resultados}

\section{Evolución de la población de lombrices}

La densidad media de la población de lombrices Eisenia andrei a lo largo del proceso de vermicompostaje fue de $452 \pm 59$ individuos $\mathrm{m}^{-2}$ (Figura $1 \mathrm{~A}$ ). El número de individuos se incrementó hasta el día 42 en un $164 \%$ ( $p=$ $0,016)$. En el día 56 la población se estabilizó manteniendo unos niveles de densidad elevados hasta el día 84, a partir del cual se apreció un importante descenso hasta el día 112 de un $37 \%(p=0,013)$. Al igual que ocurrió con la población total de lombrices el número de individuos inmaduros (Figura $1 \mathrm{~B}$ ) se incrementó de forma significativa desde el día 0 hasta el día 42 , en este caso en un $91 \%(p=0,038)$. Hasta el día 84 la densidad poblacional se estabilizó y se 
mantuvo sin cambios significativos $(p=0,742)$ registrándose a partir de este día una disminución en la población del 35\% $(p=0,014)$. Por otro lado, la densidad media de capullos fue de $104 \pm 14$ capullos $\mathrm{m}^{-2}$ (Figura $1 \mathrm{C}$ ). Su densidad aumentó de forma significativa hasta el día 84 en un $130 \%(p=0,007)$. Posteriormente se aprecia un importante descenso significativo hasta el día 112 en un 36\% ( $p=0,029)$. En cuanto a la biomasa media de la población fue de $140 \pm 19$ $\mathrm{g} \mathrm{m}^{-2}$ (Figura $1 \mathrm{D}$ ). Hasta el día 70 se incrementó de forma significativa en un 265\% ( $p<0,0001)$. A partir de ese momento y durante los últimos 42 días del proceso se produce un descenso importante de un $36 \%(p=0,01)$.

A

B
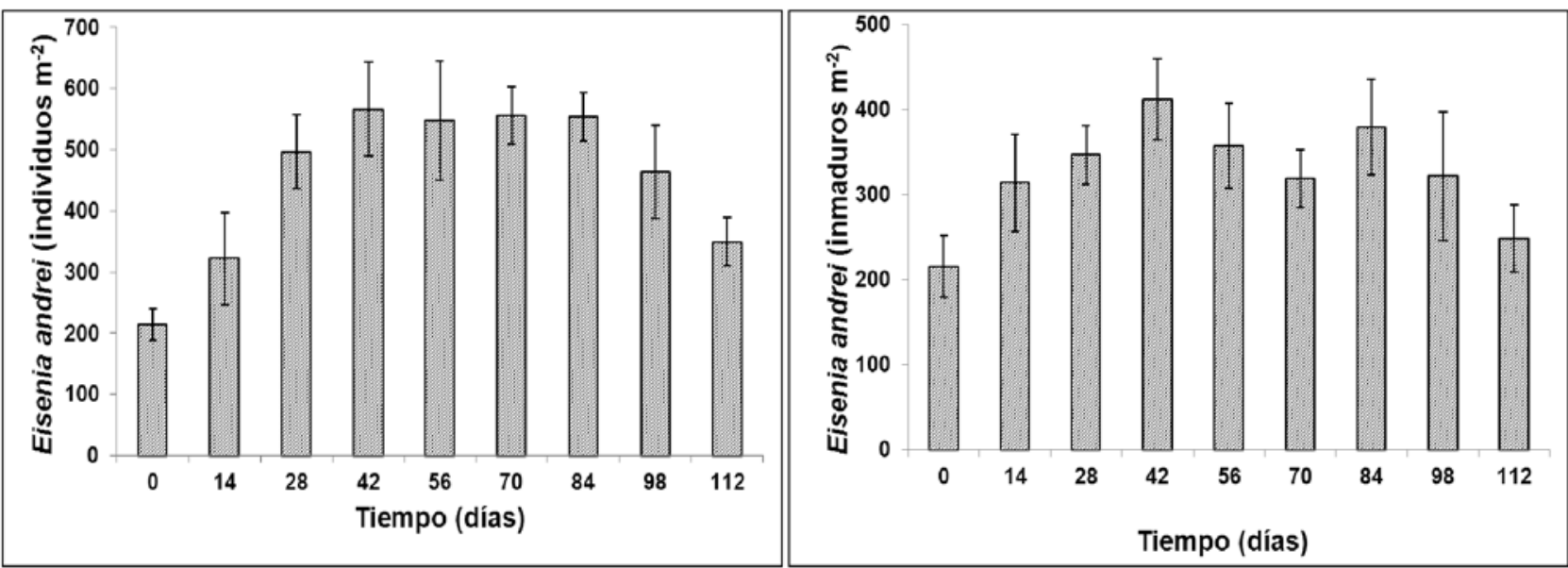

C

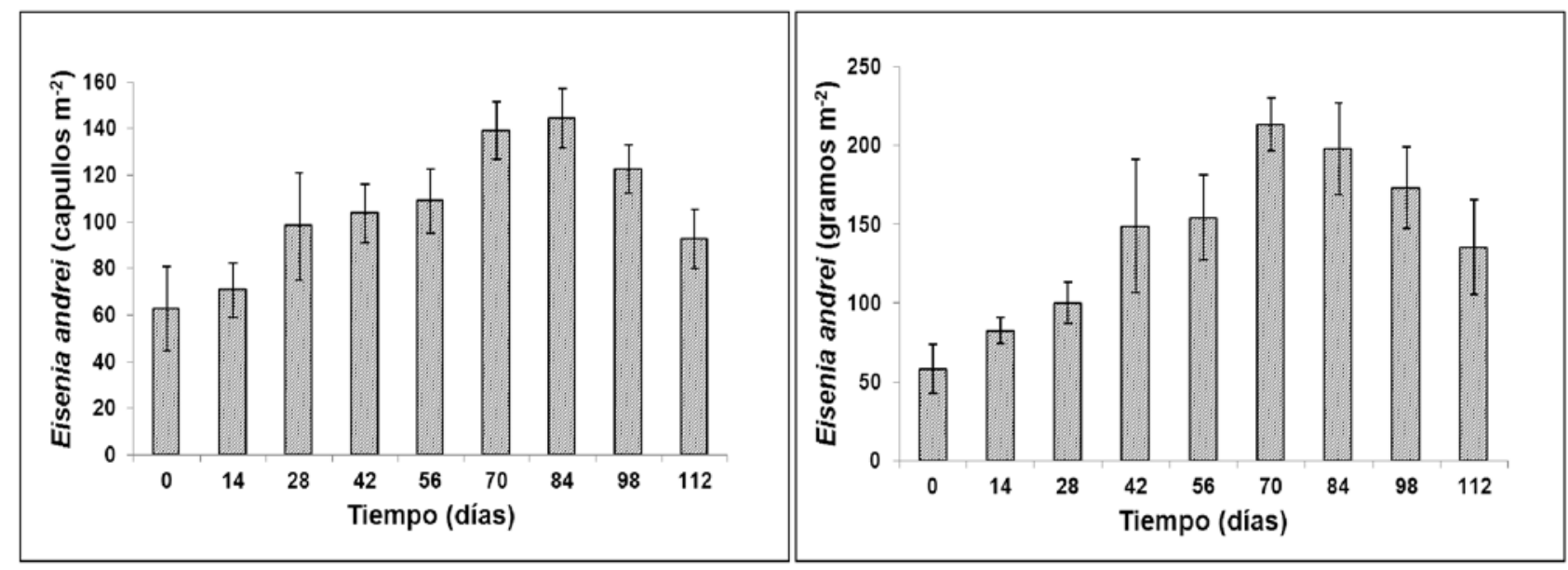

Figura 1.- Cambios en la densidad poblacional total (A) densidad de individuos inmaduros, (B), densidad de capullos (C) y biomasa (D) de lombrices (Eisenia andrei) durante el proceso de vermicompostaje del bagazo de uva. Los valores se corresponden con la media \pm el error estándar $(n=5)$

Evolución de las principales características físicas, químicas y biológicas del bagazo de uva durante su vermicompostaje

Como se puede comprobar en la Tabla 1 , el $\mathrm{pH}$ inicial fue de $4,36 \pm 0,04$ y aumentó de forma clara en un $91 \%$ hasta el día $56(p<0,0001)$. A partir de ese momento y hasta el día $112 \mathrm{el} \mathrm{pH}$ se hace significativamente menor, disminuyendo en un $13 \%$ hasta alcanzar un valor de 7,10 $\pm 0,03$ en el vermicompost final obtenido $(p=0,001)$. La conductividad eléctrica del bagazo de uva inicial fue de $1,34 \pm 0,15 \mathrm{mS} \mathrm{cm}$ y se detectó un importante descenso significativo hasta el día 56 en un $63 \%(p<0,0001)$, a partir del cual continuó disminuyendo pero de manera menos acusada finalizando con un valor de 0,27 $\pm 0,01 \mathrm{mS} \mathrm{cm} \mathrm{(} p=0,006)$.

El contenido de materia de orgánica del bagazo de uva fue de $91,21 \pm 0,30 \%$ y se mantuvo sin cambios significativos hasta el día 56, reduciéndose posteriormente en un $18 \%$ hasta el final del experimento con un contenido final de $74,98 \pm 0,34 \%(p<0,0001)$. 


\begin{tabular}{|c|c|c|c|}
\hline Características Físicas, químicas y biológicas & Día 0 & Día 56 & Día 112 \\
\hline $\mathrm{pH}$ & $4,36 \pm 0,04$ & $8,17 \pm 0,06$ & $7,1 \pm 0,003$ \\
\hline Conductividad Eléctrica $\left(\mathrm{mS} \mathrm{cm}^{-1}\right)$ & $1,34 \pm 0,15$ & $0,49 \pm 0,01$ & $0,27 \pm 0,009$ \\
\hline Materia orgánica (\% peso seco) & $91,21 \pm 0,30$ & $92,29 \pm 0,71$ & $74,98 \pm 0,34$ \\
\hline Carbono total $\left(\mathrm{g} \mathrm{kg}^{-1}\right.$ peso seco) & $484,23 \pm 1,60$ & $535,03 \pm 2,48$ & $375,96 \pm 1,47$ \\
\hline Nitrógeno total $\left(\mathrm{g} \mathrm{kg}^{-1}\right.$ peso seco) & $20,19 \pm 0,62$ & $20,56 \pm 0,95$ & $29,63 \pm 0,13$ \\
\hline Relación $\mathrm{C} / \mathrm{N}$ & $24,02 \pm 0,72$ & $26,12 \pm 1,14$ & $12,68 \pm 0,07$ \\
\hline Fósforo total ( $\mathrm{g} \mathrm{kg}^{-1}$ peso seco) & $4,03 \pm 0,08$ & $2,80 \pm 0,1$ & $8,36 \pm 0,32$ \\
\hline Potasio total $\left(\mathrm{g} \mathrm{kg}^{-1}\right.$ peso seco) & $30,46 \pm 0,56$ & $16,13 \pm 0,39$ & $11,40 \pm 0,65$ \\
\hline Actividad microbiana $\left(\mathrm{mg} \mathrm{O} 2 . \mathrm{kg}^{-1} \mathrm{MO} . \mathrm{h}^{-1}\right)$ & $122,76 \pm 0,02$ & $138,76 \pm 0,19$ & $42,93 \pm 0,005$ \\
\hline Lignina $\left(\mathrm{g} \mathrm{kg}^{-1}\right.$ peso seco) & $516,32 \pm 9,56$ & $495,76 \pm 8,96$ & $323,54 \pm 2,36$ \\
\hline Celulosa $\left(\mathrm{g} \mathrm{kg}^{-1}\right.$ peso seco) & $225,3 \pm 10,39$ & $204,53 \pm 6,77$ & $58,26 \pm 10,48$ \\
\hline Hemicelulosa ( $\mathrm{g} \mathrm{kg}^{-1}$ peso seco) & $100,6 \pm 1,39$ & $90,83 \pm 0,89$ & $30,56 \pm 0,54$ \\
\hline
\end{tabular}

Tabla 1.- Cambios en las características físicas, químicas y biológicas del bagazo de uva durante el proceso de vermicompostaje. Los valores se corresponden con la media \pm el error estándar $(n=3)$

El contenido de carbono total del bagazo inicial fue de $484,23 \pm 1,60 \mathrm{~g} \mathrm{~kg}^{-1}$ peso seco. Aumentó ligeramente hasta el día 56, para luego disminuir de forma significativa en un $30 \%$ hasta alcanzar $375,96 \pm 1,47 \mathrm{~g} \mathrm{~kg}^{-1}$ peso seco al final del experimento $(p=0,001)$. Sin embargo, el nitrógeno total presentó unos valores iniciales de 20,19 $\pm 0,62 \mathrm{~g} \mathrm{~kg}^{-1}$ peso seco aumentando en un $47 \%$ hasta la obtención del vermicompost final con un contenido de 29,63 $\pm 0,13 \mathrm{~g} \mathrm{~kg}^{-1}$ peso seco $(p=0,008)$. De esta manera la relación $\mathrm{C} / \mathrm{N}$ inicial fue de $24,02 \pm 0,72$, se mantuvo sin cambios significativos hasta el día 56 para luego disminuir significativamente en un $51 \%$ con una relación de $12,68 \pm 0,07$ en el último día de vermicompostaje $(p=0,006)$

El contenido de fósforo total en el bagazo inicial fue de 4,03 $\pm 0,08 \mathrm{~g} \mathrm{~kg}^{-1}$ peso seco. Tras los 112 días de vermicompostaje aumentó significativamente en un $99 \%$ y finalizó con un contenido de 8,36 $\pm 0,32 \mathrm{~g} \mathrm{~kg}^{-1}$ peso seco $(p=0,008)$. En cambio, el potasio total que presentaba un contenido $30,46 \pm 0,56 \mathrm{~g} \mathrm{~kg}^{-1}$ peso seco en el bagazo inicial disminuyó significativamente durante todo el proceso en un $81 \%$ finalizando en el vermicompost final con un valor de $11,4 \pm 0,65 \mathrm{~g} \mathrm{~kg}^{-1}$ peso seco $(\mathrm{p}=0,037)$.

La actividad microbiana del bagazo de uva inicial fue de $122,76 \pm 0,02 \mathrm{mg} \mathrm{O}_{2} \mathrm{Kg}^{-1} \mathrm{MO} \mathrm{h}^{-1}$. Aumentó ligeramente hasta el día $56(p<0,0001)$ y disminuyó posteriormente en un $69 \%$ para alcanzar en el vermicompost final una tasa de respiración de 42,93 $\pm 0,005 \mathrm{mg} \mathrm{O}_{2} \mathrm{Kg}^{-1} \mathrm{MO} \mathrm{h}^{-1}(p<0,0001)$.

En cuanto a las fibras analizadas el contenido de celulosa y hemicelulosa del bagazo inicial fue $225,3 \pm 10,39$ y 100,6 \pm $1,39 \mathrm{~g} \mathrm{~kg}^{-1}$ peso seco respectivamente; su concentración varió de forma significativa tras 112 días de vermicompostaje finalizando con un contenido de celulosa de $58,26 \pm 10,48 \mathrm{~g} \mathrm{~kg}^{-1}$ peso seco $(\mathrm{p}=0,011)$ y un contenido de hemicelulosa de 30,56 $\pm 0,54 \mathrm{~g} \mathrm{~kg}^{-1}$ peso seco $(p<0,0001)$. La lignina en el bagazo inicial presentó un valor $516,32 \pm 9,56 \mathrm{~g} \mathrm{~kg}^{-1}$ peso seco y tras los 112 días de vermicompostaje finalizó con un contenido de 323,54 $\pm 2,36$ $\mathrm{g} \mathrm{kg}^{-1}$ peso seco $(p<0,0001)$.
Evolución del índice de polifenoles totales (IPT) en el bagazo de uva y semillas a lo largo del proceso de vermicompostaje

El contenido de polifenoles en el bagazo de uva inicial (Figura $2 \mathrm{~A}$ ) fue de $58 \pm 5 \mathrm{mg} \mathrm{GAE} \mathrm{g}^{-1}$ bagazo seco. La concentración disminuyó considerablemente a lo largo del tiempo de vermicompostaje $(p<0,0001)$. Los resultados del test a posteriori HSD de Tukey indican que la mayor diferencia en la cantidad de polifenoles presentes en el bagazo de uva se da entre los días 7 y 112 con una disminución del 98\%, manteniéndose en las últimas semanas en unos niveles muy bajos en comparación con los presentados antes del inicio del vermicompostaje y alcanzando en el producto final una concentración de 1,37 $\pm 0,07 \mathrm{mg} \mathrm{GAE} \mathrm{g}^{-1}$ bagazo seco.

En el caso de las semillas aisladas (Figura 2 B) la concentración inicial de polifenoles fue de $70 \pm 1 \mathrm{mg} \mathrm{GAE} \mathrm{g}^{-1}$ semilla seca. A lo largo del tiempo de vermicompostaje la concentración disminuyó de forma significativa $(p<0,0001)$. El test a posteriori HSD de Tukey indica que la mayor diferencia en la concentración de polifenoles se da entre los días 0 y 112, entre los cuales los valores obtenidos se redujeron en un $84 \%$ alcanzando en el día 112 una concentración de $11 \pm 0,1 \mathrm{mg} \mathrm{GAE} \mathrm{g}^{-1}$ semilla seca.

Evolución de los polifenoles individuales en las semillas de uva a lo largo del proceso de vermicompostaje

Los principales polifenoles identificados en las semillas aisladas a una longitud de onda de $280 \mathrm{~nm}$ fueron ácido gálico, catequina y epicatequina y a una longitud de onda de $350 \mathrm{~nm}$ se identificaron las quercetinas (Figura 3). Al comienzo del proceso, la concentración del ácido gálico fue

de $240 \pm 3 \mathrm{mg} \mathrm{kg}^{-1}$ semilla seca. Su concentración descendió de forma significativa en un $50 \%$ a lo largo de los 112 días y finalizó con un valor de $120 \pm 10 \mathrm{mg} \mathrm{kg}^{-1}$ semilla seca $(p<0,0001)$. La catequina en las muestras de semillas 
del día 0 registró una concentración de $4790 \pm 120 \mathrm{mg} \mathrm{kg}^{-1}$ semilla seca y disminuyó también de manera significativa hasta el día 112 en un $49 \%$, alcanzando una concentración final de $2420 \pm 120 \mathrm{mg} \mathrm{g}^{-1}$ semilla seca ( $p<0,0001$ ). En el caso de la epicatequina su concentración en el día 0 fue de $2510 \pm 60 \mathrm{mg} \mathrm{kg}^{-1}$ semilla seca y disminuyó hasta el día 112 de manera significativa en un $52 \%$, finalizando el proceso

A

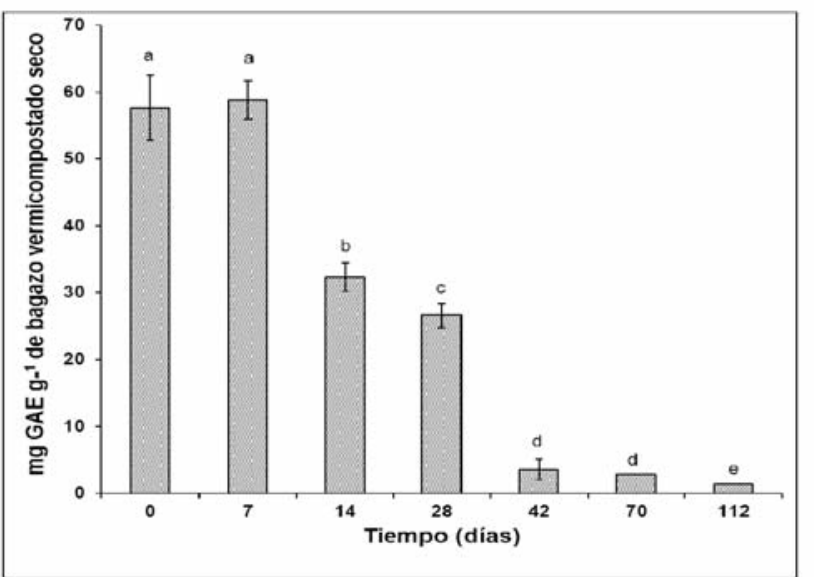

Figura 2.- Cambios en la concentración de polifenoles totales ( $\mathrm{mg} / \mathrm{GAE} \mathrm{g}^{-1}$ peso seco) en las muestras de bagazo vermicompostado (A) y semillas (B) analizadas. Las letras diferentes representan diferencias significativas (test HSD de Tukey, $p<0,05$ ). Los valores se corresponden con la media \pm el error estándar $(n=5)$

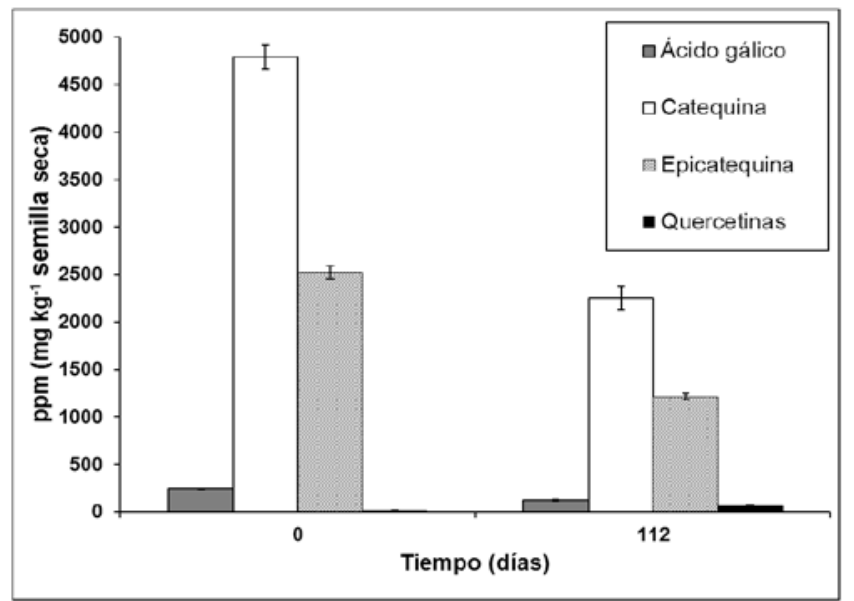

Figura 3.- Cambios en la concentración de ácido gálico, catequina, epicatequina y quercetinas en las semillas durante el proceso de vermicompostaje. Los valores se corresponden con la media \pm el error estándar $(n=5)$ con una concentración de $1210 \pm 30 \mathrm{mg} \mathrm{kg}^{-1}$ semilla seca $(p<0,0001)$. En cuanto a la concentración de quercetinas se registró un valor inicial de $10 \pm 0,5 \mathrm{mg} \mathrm{kg}^{-1}$ semilla seca. En este caso la concentración aumentó de forma significativa a del proceso una concentración final de $59 \pm 3 \mathrm{mg} \mathrm{kg}^{-1}$ semilla seca $(p<0,0001)$. lo largo del tiempo en un $490 \%$ hasta alcanzar en el día 112

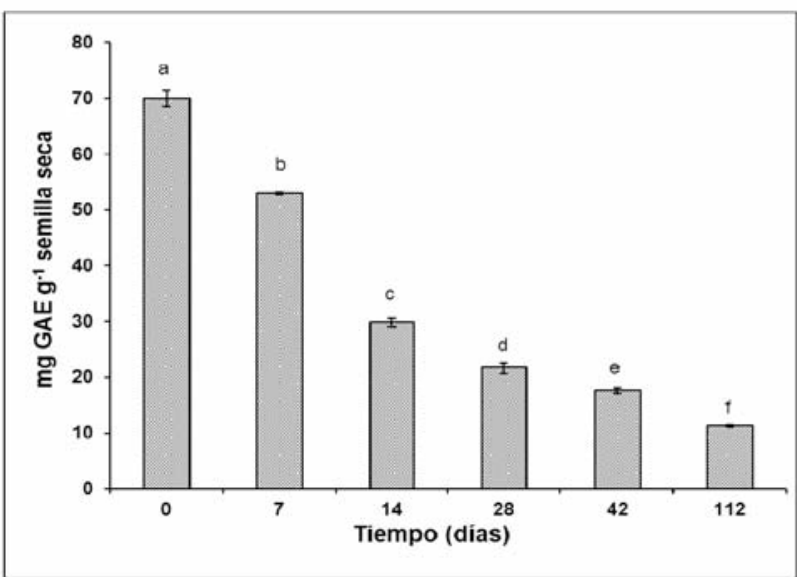

\section{Discusión}

Los resultados del proceso de vermicompostaje muestran una buena actividad de las lombrices de tierra Eisenia andrei durante el experimento. La fase activa finalizó entre los días 42 y 70 de vermicompostaje, momento a partir del cual la densidad y biomasa de la población empieza a decrecer. En esta fase la concentración de nutrientes es elevada y teniendo en cuenta que el bagazo es una fuente de nitrógeno importante aumentan significativamente el

peso y la tasa reproductiva de las lombrices (Evans \& Guild 1948). En la etapa de maduración, durante las semanas siguientes, disminuyeron las partes del bagazo más fácilmente biodegradables por las lombrices, que pasaron a ocupar un papel secundario; y la densidad de individuos, la densidad de capullos y la biomasa decrecieron considerablemente. Durante este periodo son los microorganismos los que asumen el control del vermicompostaje en las etapas finales del proceso (Domínguez et al. 2010). 
En cuanto a los parámetros físicos, químicos y biológicos estudiados, el $\mathrm{pH}$ aumenta en las etapas iniciales del proceso. El pH está relacionado con los niveles de nitrógeno amoniacal, así un aumento de éste suele conllevar un aumento de $\mathrm{pH}$ y viceversa (Atlas \& Bartha 2002), estabilizándose en este caso en las etapas finales del experimento. Según Edwards (1998), el pH óptimo para el desarrollo vegetal se sitúa entre 5,5 y 6 , valores cercanos a los obtenidos; al mismo tiempo una concentración de sales elevada puede limitar el desarrollo vegetal (Brownell \& Schneider 1985). En este caso la conductividad eléctrica registró una disminución progresiva y se estabilizó en valores muy por debajo de $8 \mathrm{mS} \mathrm{cm}^{-1}$, que es el límite de supervivencia para las lombrices (Edwards 1988). A lo largo del vermicompostaje, una fracción de la materia orgánica contenida en el bagazo se mineraliza (Hartestein \& Hartestein 1981; Mitchell et al. 1982; Gómez-Brandón et al. 2010), de esta manera, descienden las concentraciones de materia orgánica y carbono total, y por lo tanto la relación $\mathrm{C} / \mathrm{N}$, cuyo valor en este experimento es próximo al óptimo para considerar un buen grado de madurez, en torno a un valor de 12 (Jiménez \& García 1989). Estos valores son comparables a los observados en un experimento previo con bagazo (Gómez-Brandón et al. 2010) donde se registra la misma tendencia tras la fase activa del vermicompostaje. Como consecuencia de la mineralización de la materia orgánica, se favorece la concentración de nutrientes como el nitrógeno y fósforo en las etapas finales del proceso, aunque en el caso del potasio desciende de manera progresiva, posiblemente debido a que es un nutriente muy móvil y fácilmente lixiviado por las acciones de riego durante el vermicompostaje.

La actividad microbiana, ampliamente relacionada con todos los procesos estudiados se incrementó durante la fase activa, posiblemente como consecuencia de la alta disponibilidad de compuestos fácilmente biodegradables y por la acción excavadora de las lombrices de tierra que favorecen esta actividad. La disminución de su actividad tiene lugar en las últimas semanas del proceso, al disminuir las mejor degradables por los microorganismos. La actividad microbiana influye directamente en el contenido de fibras del bagazo. Así, se observó una reducción de lignina, hemicelulosa y celulosa a lo largo del tiempo y una disminución significativa durante la fase de maduración. En el caso de la lignina, Gomez-Brandón et al. (2010) encuentran que no se producen cambios significativos durante los primeros 15 días del proceso de vermicompostaje, posiblemente se debe a que el proceso de degradación de la lignina es lento y requiere una buena actividad microbiana a largo plazo. El bagazo de uva está constituido por un $18,2 \%$ de celulosa; $8 \%$ de hemicelulosas y un $56,7 \%$ de lignina (Cruz et al. 2004). Son moléculas recalcitrantes y solamente degradadas por microorganismos en condiciones aerobias (Dommergues \& Mangenot 1970). De esta manera, se observó un descenso en su concentración lento pero constante, en parte debido a la actividad microbiana registrada durante todo el proceso.

Los polifenoles totales muestran una disminución progresiva en su concentración a lo largo del tiempo. En la literatura científica se ha demostrado que de modo general, la actividad de las lombrices de tierra, promueve el incremento de las poblaciones microbianas (Edwards \& Bohlen 1996), los cuales están especializados en degradar estos compuestos más complejos (Domínguez et al. 2010). En el caso de las semillas asiladas los polifenoles se encuentran en mayor concentración que en el bagazo vermicompostado. Esto se debe a que no pueden ser degradadas por las lombrices y por lo tanto se hacen menos disponibles para el ataque de los microorganismos. Las semillas son por lo tanto interesantes como una fuente de polifenoles de alto interés industrial y comercial.

Durante el vermicompostaje se produce una separación mecánica en el bagazo de uva vermicompostado, las partes del bagazo más fácilmente digeribles por las lombrices adquieren una granulometría más fina y ocupan las zonas inferiores. Sobre la superficie quedan principalmente las semillas, los tallos y las pieles más duras que son la fracción más recalcitrante del bagazo de uva (Berg \& McClaugherty 2003) y de mayor concentración polifenólica. En las últimas semanas de la fase activa esta separación ya tuvo lugar y el óptimo de recogida de estas semillas manualmente se da aproximadamente entre los días 28 y 42 antes de que la concentración polifenólica continúe disminuyendo. La separación natural que tiene lugar durante todo el proceso optimizó a su vez la realización de un tamizado de las muestras a partir del día 42. El tamizado realizado a través de $1 \mathrm{~mm}$ de luz de malla permitió aislar completamente las semillas del vermicompost producido, permitiendo la obtención de la fuente más rica de polifenoles y eliminando así la parte del bagazo vermicompostado de mayor fitotoxicidad. En el vermicompost final la eliminación de estas semillas y a su vez la descomposición de estos compuestos fitotóxicos indica un buen grado de madurez (Wu et al. 2000), un hecho de suma importancia ya que el vermicompost inmaduro puede afectar negativamente al desarrollo de cultivos (Hirai et al. 1983; He et al. 1995). De esta manera se obtiene un producto estabilizado y maduro con grandes posibilidades de utilización en agricultura.

Por otro lado, los resultados muestran que a una longitud de onda de $280 \mathrm{~nm}$ los principales polifenoles identificados en las semillas fueron ácido gálico, catequina y epicatequina, siendo la catequina el compuesto más abundante. En el caso de la detección a $350 \mathrm{~nm}$, se observaron diferentes tipos de quercetinas como componentes mayoritario, los cuales son flavonoles abundantes en los vegetales. Las concentraciones de los polifenoles individuales siguen la misma tendencia que el índice polifenólico total por las acciones degradadores de lombrices y microorganismos. En el caso de las quercetinas, se detectó un incremento de concentración importante. Los datos se obtuvieron por suma de distintos tipos de quercetinas, que pudieron evolucionar de manera diferente. Para explicar este comportamiento desigual con respecto a los demás polifenoles, se requiere una identificación precisa de las distintas quercetinas individuales, previa reoptimización del método cromatográfico con este fin.

Diversos estudios corroboran los datos obtenidos para polifenoles, ya que se ha demostrado que las frutas, como las uvas, constituyen una de las principales fuentes de compuestos polifenólicos, especialmente ácidos benzoicos, 
ácidos cinámicos, antocianidinas, flavonoles, catequinas y taninos (García-Alonso et al. 2002), los cuales se mantienen en el bagazo de uva y de manera elevada en las semillas. Además, la vinificación en blanco aumenta la proporción de polifenoles que se mantienen en el residuo. Tanto la catequina como la epicatequina, que son los principales polifenoles en semillas, tienen una capacidad secuestradora de radicales libres 10 veces superior a la de L-ascorbato y $\beta$-caroteno (Yilmaz 2005). También reducen la presión arterial (García- Salas et al. 2010) y presentan actividad inhibitoria de enzimas clave involucradas en el ciclo celular, inducen apoptosis en diferentes líneas celulares e inhiben la expresión de ciertos genes relacionados con tumores. Todas estas características hacen de las semillas un producto del bagazo de especial interés y con grandes aplicaciones industriales y comerciales.

\section{Conclusión}

El bagazo de uva, una vez tratado mediante un proceso de vermicompostaje constituye un producto maduro, estabilizado y con grandes posibilidades de uso como enmienda orgánica para campos de cultivo. Simultáneamente, el proceso de vermicompostaje facilita el aislamiento de las semillas del bagazo y las convierte en una fuente de polifenoles bioactivos con aplicaciones interesantes para diferentes actividades industriales.

\section{Bibliografía}

Atlas, R.M \& Bartha, R. (2002). Ecología microbiana y Microbiología ambiental. Addison Wesley, Madrid.

Berg, B. \& McClaugherty, C. (2003). Plant Litter: Decomposition, Humus Formation, Carbon Sequestration. Springer-Verlag Berlin Heidelberg, New York.

Bertran, E., Sort, X., Soliva, M. \& Trillas, I. (2004). Composting winery waste: sludges and grape stalks. Bioresource Technology. 95; 203-208.

Brownell, K.H. \& Schneider, R.W. (1985). Roles of matric and osmoticcomponents of water potential and their interaction with temperature in the growth of Fusarium oxysporum in synthetic media and soil. Phytopathology.75: 53-57.

Bouché, M. (1972). Lombriciens de France. Ecologie et systématique. Annales de Zoologie et Ecologie Animale, Numéro hors-série. Institut National de la Recherche Agronomique, Paris.

Bustamante, M.A. (2007). Compostaje de los residuos generados en la inductria vinícola y alcoholes. Valorización agronómica de los materiales obtenidos. Tesis doctoral, Universidad Miguel Hernández de Elche.

Cegarra, J. \& Paredes C. (2007). Residuos agroindustriales. En: J. Moreno \& R. Moral (Eds.). Compostaje. Ediciones Mundi-Prensa, Madrid. 519-553
Cruz, J.M., Domínguez, H. \& Parajo, J.C. (2004). Assessment of the production of antioxidants from winemaking waste solids. Journal of Agricultural and Food Chemistry. 56: 5612-5620.

Dell'Agli, M., Buscialà, A., \& Bosisio, E. (2004). Vascular effects of wine polyphenols. Cardiovascular Research. 63, 4: 593-602.

Dommergues, Y. \& Mangenot, F. (1970). Écologie microbienne du sol. Masson et Cie. París.

Domínguez, J. (2004). State of the art and new perspectives on vermicomposting research. En: C.A. Edwars (Ed.). Earthworm ecology. $2^{\text {nd }}$ d. CRC Press, Boca Raton. 401424.

Domínguez, J., Aira, M., Gómez-Brandón, M. (2010). Vermicomposting: earthworms enhance the work of microbes. En: H. Insam et al. (Eds), Microbes at work: from wastes to resources. Springer-Verlag, Berlin Heildelberg. 93-114.

Domínguez, J. \& Edwars C.A. (2010). Biology and ecology of earthworm species used for vermicomposting. En: C.A. Edwars et al. (Eds.). Vermiculture technology: Earthworms, organic waste and environmental management. CRC Press. Boca Raton, Florida. 25-37.

Edwards, C.A. \& Bohlen, P.J. (1996). Biology and ecology of earthworms. Chapman and Hall, London.

Edwards, C.A. (1988). Breakdown of animal, vegetable and industrial organic wastes by earthworms. En: C.A. Edwards \& E.F. Neuhauser (Eds.). Earthworms in waste and enviromental management. SPB Academic Publishing, The Hague. 21-31

Evans, A.C. \& Guild, W.J.Mc.L. (1948). Studies on the relationships between earthworms and soil fertility. IV. On the life cycles of some British Lumbricidae. Annals of Applied Biology. 35: 471-484.

García-Alonso, J., Periago, M.J, Vidal Guevara, M.L., Cantos, E. (2002). Evaluación de las propiedades antioxidantes en concentrados de uva frutas rojas. Anales de Veterinaria (Murcia). 18: 103-114.

García-Salas, P., Morales-Soto, A., Segura-Carretero, A. \& Fernandez-Gutiérrez, A. (2010).

Phenolic-compound-extraction systems for fruit and vegetable samples. Molecules. 15: 8813-8826.

Gharras, H. (2009). Polyphenols: Food sources, properties and applications - A review. International Journal of Food Science and Technology. 44, 12: 2512-2518.

Goering, H. K \& Van Soest, P. J. (1970). Forage fiber analyses (apparatus, reagents, procedures, and some applications). Agriculture Handbook. 379.

Gómez-Brandón, M., Lazcano, C., Lores, M. \& Domínguez, J. (2010). Papel de las lombrices de tierra en la degradación del bagazo de uva: efectos sobre las características químicas y la microflora en las primeras etapas del proceso. Acta Zoológica Mexicana (n.s.), Número Especial 2: $397-$ 408. 
Hartenstein, F., Hartenstein, E. \& Hartenstein, R. (1981). Gut load and transit time in the earthworm Eisenia foetida. Pedobiologia. 22: 5-20.

He, X.T., Logan, T.J. \& Traine, S.J. (1995). Physical and chemical characteristics of selected U.S. municipal solid waste compost. Journal of Environmental Quality. 24: 543552.

Hirai, M.F., Chanyasak, V., Kubota, M. (1983). A standard measurement for compost maturity. Biocycle. 24: 54-56.

Inbar, J. \& Chet, Y. (1991). Detection of chitinolytic activity in the rhizosphere using image analysis. Soil Biology \& Biochemistry. 23: 239-242.

Jiménez, E. \& García, V. (1989). Evaluation of city refuse compost maturity: a review. Biological wastes. 27: 115-142.

Maier, T., Schieber, A., Kammerer, D.R., Carle, R. (2009). Residues of grape (Vitis vinifera L.) seed oil production as a valuable source of phenolic antioxidants. Food Chemistry. 112, 3: 551-559.

Mitchell, M.J., Parkinson, C.M., Hamilton, W.E. \& Dindal, D.L. (1982). Role of the earthworm Eisenia foetida, in affecting organic matter decomposition in microcosms of sludge-amended soil. Journal of Applied Ecology. 19: 805812.
Quideau, S., Deffieux, D., Douat-Casassus, C. \& Pouységu, L. (2011). Plant polyphenols: Chemical properties, biological activities, and synthesis. Angewandte Chemie International Edition. 50, 3: 586- 621.

Rice-Evans, C. (2001). Flavonoid antioxidants. Current Medicinal Chemistry. 8: 797-807.

Singleton, V.L., Rossi, J.A. Jr. (1965). Colorimetry of total phenolics with phosphomolybdic-phosphotungstic acid reagents. American Journal of Enology and Viticulture. 16; 144-158.

Usaquén-Castro, X., Martínez-Rubio, M., Aya-Baquero, H. \& González-Martínez, G. (2006). Ultrasound-assisted extraction of polyphenols from red-grape (Vitis vinifera) residues. IUFOST.

Wu, L., Ma, L.Q., Martinez, G.A. (2000). Comparison of methods for evaluating stability and maturity of biosolids compost. Journal of Environmental Quality. 29: 424-429.

Yilmaz, M. (2005). The effects of rosiglitazone and metformin on oxidative stress and homocysteine levels in lean patients with polycystic ovary sundrome. Human Reproduction. 20: 333-340. 